\title{
Giraffidae (Mammalia) de la fin du Néogène de la République de Macédoine (ARYM)
}

\author{
Denis GERAADS \\ CNRS UPR 2147, 44 rue de l'Amiral Mouchez, F-75014 Paris (France) \\ denis.geraads@evolhum.cnrs.fr
}

\author{
MOTS CLÉS \\ Mammalia, \\ Giraffidae, \\ Miocène supérieur, \\ Pliocène, \\ Macédoine, \\ ARYM.
}

KEY WORDS

Mammalia,

Giraffidae,

upper Miocene,

Pliocene,

Macedonia,

FYROM.
Geraads D. 2009. - Giraffidae (Mammalia) de la fin du Néogène de la République de Macédoine (ARYM). Geodiversitas 31 (4): 893-908.

\section{RÉSUMÉ}

Les fouilles menées dans plusieurs gisements de la République de Macédoine (ARYM) au cours des dernières décennies ont mis au jour de riches faunes mammaliennes, parmi lesquelles quelques pièces significatives de Giraffidae. Un crâne presque complet et non déformé du Miocène supérieur de Kirokuçuk est le meilleur spécimen connu de Bohlinia attica; il confirme la proche parenté de ce taxon et de Giraffa, même s'il est plus primitif que les formes africaines de ce dernier genre. Helladotherium est représenté par des éléments de crânes et os des membres. On retrouve cette association de taxons en Bulgarie et à Pikermi en Grèce. Des éléments d'un crâne, incluant la plus grande partie d'une corne postérieure, d'âge probablement pliocène, sont attribués à une nouvelle espèce de Sivatherium, caractérisée par ses cornes postérieures gigantesques, impliquant des échanges fauniques entre l'Europe du Sud, l'Afrique et l'Asie du Sud à cette époque.

\section{ABSTRACT}

Giraffidae (Mammalia) from the late Neogene of the Republic of Macedonia (FYROM).

Excavations undertaken in several sites of the Republic of Macedonia (FYROM) during the last decades have yielded rich mammalian faunas, including some significant giraffid specimens. An almost complete and uncrushed skull from the upper Miocene of Kirokuçuk is the best known specimen of Bohlinia attica; it confirms that this taxon is closely related to Giraffa, even though it is more primitive than the African forms of the latter genus. Helladotherium is represented by cranial and limb-bone remains. The association of these two genera is also found in Bulgaria and at Pikermi in Greece. Cranial elements, including most of a posterior horn, are assigned to a new species of Sivatherium, probably of Pliocene age, and characterized by its huge posterior horns. Its presence implies faunal connections between Southern Europe, Southern Asia and Africa at that time. 


\section{INTRODUCTION}

Bien que connus depuis le début du Xxe siècle, les mammifères du Miocène supérieur de ce qui constitue aujourd'hui la République de Macédoine (aussi appelée ARYM, Fig. 1), n'ont fait l'objet que de très peu d'études, parmi lesquelles les plus synthétiques sont celles de Schlosser (1921) et Ciric (1957). Au cours des dernières décennies, plusieurs nouveaux sites ont été découverts dans diverses régions du pays, et exploités sous la direction de Risto Garevski; ils sont pour la plupart concentrés dans la vallée du Vardar (= Axios en Grèce), et spécialement dans la région de Veles (= Titov Veles). Le matériel recueilli, qui fait partie de la collection Garevski conservée au Muséum d'Histoire naturelle de Skopje, reste pour l'essentiel inédit, à l'exception de quelques taxons (Garevski 1960a, b, 1974a, b, 1976, 1989; Garevski \& Zapfe 1983; Forstén \& Garevski 1989; Geraads et al. 2008). D'après ces travaux et les études en cours, tous les gisements importants (Kirokuçuk, Karaslari, Vozarci) semblent appartenir au Turolien, mais seule une étude détaillée permettra de les placer plus précisément à l'intérieur de cet étage mammalien. Les principales pièces appartenant aux Giraffidae sont décrites ci-dessous.

Par ailleurs, en 2007, ont été découverts fortuitement près de Delcevo dans le nord-est du pays, et dans un niveau probablement pliocène, des éléments très fragmentés d'un crâne de Sivatheriinae. Ce matériel a été remis en 2008 au MHNS, où j'ai pu remonter la plus grande partie d'une corne postérieure.

$\begin{array}{ll}\text { ABRÉVIATIONS } \\ \text { BMNH } & \text { Natural History Museum, Londres; } \\ \text { MHNS } & \text { Muséum d'Histoire naturelle, Skopje; } \\ \text { MNHN } & \text { Muséum national d'Histoire naturelle, Paris; } \\ \text { NHMW } & \text { Naturhistorisches Museum, Vienne; } \\ \text { PIUW } & \text { Paläontologisches Institut Universität, Vienne; } \\ \text { DAP } & \text { dimension antéro-postérieure; } \\ \text { DT } & \text { dimension transversale; } \\ \text { Ka } & \text { Karaslari; } \\ \text { KK } & \text { Kirokuçuk; } \\ \text { NKT } & \text { Nikiti } 1 ; \\ \text { TM } & \text { Toros-Menalla; } \\ \text { Voz } & \text { Vozarci. }\end{array}$

Les mesures sont en $\mathrm{mm}$. Les dents supérieures sont en majuscules, les inférieures en minuscules.

\section{SYSTÉMATIQUE}

\author{
Famille GirafFIDAE Gray, 1821 \\ Sous-famille GiraffinaE Gray, 1821
}

DiAgNose. - Cornes principales courtes, originellement indépendantes du toit crânien, mais fusionnant avec ce dernier chez l'adulte, à ossification s'accroissant avec l'âge, et finissant par être constituées d'os compact (ossicônes). Ces ossicônes sont disposés en arrière des orbites et rapprochés l'un de l'autre. Arrière-crâne non raccourci; orbites situées assez antérieurement; museau étroit, à portion prédentaire longue; dents brachyodontes, à forts styles et piliers.

J'inclus dans cette sous-famille la girafe actuelle, Giraffa camelopardalis Linnaeus, 1758 (s.l., car les recherches récentes [Brown et al. 2007] semblent montrer que plusieurs espèces ont été confondues sous ce nom), les formes fossiles africaines de Giraffa Brisson, 1762, Bohlinia attica (Gaudry \& Lartet, 1858), et Okapia Lankester, 1901. La position de ce dernier genre a plus d'une fois été débattue (Bohlin 1926; Geraads 1986; Thenius 1992), mais c'est le seul giraffidé en dehors de Bohlinia et Giraffa dont les cornes présentent les caractères cités dans la diagnose, et méritent le nom d'ossicônes; rappelons qu'en revanche une origine indépendante des os du crâne n'a jamais été établie chez les autres giraffidés fossiles, et l'hyper-ossification est assurément absente chez ces derniers (Geraads 1991). Il est probable, comme le pensait Thenius (1992), que l'adaptation de l'okapi à la forêt est secondaire, et il se pourrait qu'il partage un ancêtre relativement récent avec les girafes.

\section{Tribu GIRAFFInI Gray, 1821}

DiAGNOSE. - Ossicônes à extrémité arrondie, crâne très large au niveau de la barre post-orbitaire, orbites regardant très antérieurement pour un ruminant; crêtes des prémolaires inférieures tendant à se subdiviser; os des membres, et probablement cou, très allongés.

J'inclus dans cette tribu tous les Giraffinae sauf Okapia. Ces acceptions de la sous-famille des Giraffinae et de la tribu des Giraffini sont plus proches de celles traditionnellement acceptées que de celles récemment proposées par Solounias (2007).

\section{Genre Bohlinia Matthew, 1929}

ESPÈCE TYPE. - Orasius speciosus Wagner, 1861 (par désignation originale). Ce nom est un synonyme récent de Camelopardalis attica Gaudry \& Lartet, 1856. 
TABLEAU 1. - Mensurations crâniennes (en mm) de Bohlinia attica (Gaudry \& Lartet, 1856) comparées à celles de Giraffa jumae Leakey, 1967 (National Museums of Kenya, Nairobi) et à celles de la moyenne d'un échantillon ( $N=18)$ de G. camelopardalis Linnaeus, 1758 actuel (valeurs extrêmes entre parenthèses). Notes: 1, valeur estimée d'après photo ou figure; 2, Bohlin 1926.

\begin{tabular}{lccccc}
\hline & Kirokuçuk A & Kirokuçuk B & Pikermi $^{2}$ & G. jumae & actuel \\
\hline Largeur maximum bi-orbitaire & 260 & - & - & 335 & $269(245-325)$ \\
Largeur bi-condylienne & 101 & - & - & 109 & $105(91-113)$ \\
Largeur bi-mastoïdienne & 152 & - & - & 163 & $162(141-190)$ \\
Longeur condyle - avant de P2 & 357 & c. 360 & 3551 & 405 & $358(313-405)$ \\
Longeur condyle - arrière de M3 & 225 & c. 230 & 2101 & 227 & $222(174-260)$ \\
Longeur crête occipitale - orbite & - & 220 & 2101 & 265 & $240(174-260)$ \\
\hline
\end{tabular}

Bohlinia attica (Gaudry \& Lartet, 1856)

Camelopardalis attica Gaudry \& Lartet, 1856: 274.

Orasius atticus - Bohlin, 1926: 123.

TYPE. - Geraads (1979: 378) désigna comme lectotype un spécimen n'appartenant pas à la série type, et ce choix ne peut donc pas être retenu (Gentry 2003: 344). Aucun lectotype ne peut actuellement être désigné car la série type, non figurée, a par la suite été mélangée à d'autres spécimens.

MATÉRIEL ÉTUdié. - Crâne presque complet et non déformé de Kirokuçuk (crâne A, Fig. 2A, B), auquel ne manquent que la partie antérieure du museau, la crête occipitale et la plus grande partie des ossicônes; crâne presque complet du même site (crâne B), auquel manquent les pointes des ossicônes, l'extrémité du museau et les condyles occipitaux, et très incomplètement dégagé de son plâtre. De Karaslari, proviennent un maxillaire, $\mathrm{Ka}-2603$, et quelques os des membres, pour la plupart non dégagés de leurs plâtres. Tout ce matériel est conservé au MHNS.

\section{LOCALITÉ TYPE. - Pikermi, Grèce (Turolien).}

EXTENSION STRATIGRAPHIQUE ET GÉOGRAPHIQUE. Vallésien final au Turolien final de la Province balkanoiranienne (Spassov \& Geraads 2004).

DiAGNOSE. - Une seule paire de cornes situées au-dessus de la barre post-orbitaire, frontal modérément pneumatisé; dents petites par rapport au crâne, prémolaires de lait primitives; os des membres graciles. Tous ces caractères sont primitifs par rapport à ceux de Giraffa.

\section{DESCRIPTION}

En vue dorsale (Fig. 2B), le crâne A frappe par la disproportion entre un museau et une région pariétale étroits et un frontal très élargi, spécialement au niveau des bords postérieurs des orbites

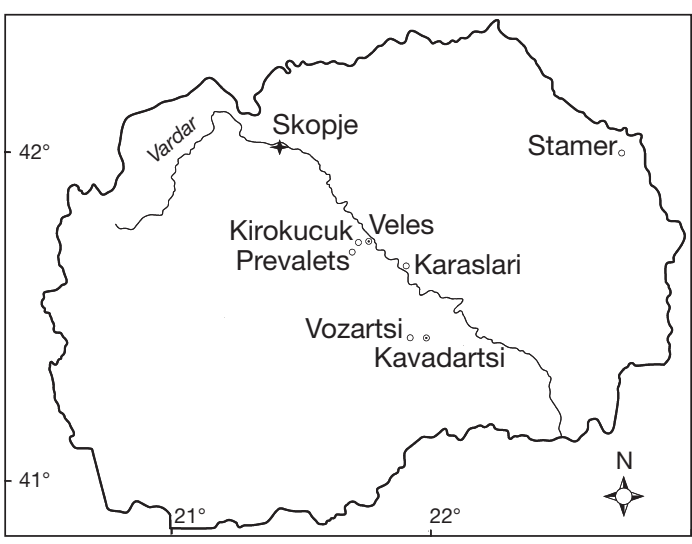

FIG. 1. - Localisation des sites (République de Macédoine) mentionnés dans l'article. Carte N. Spassov.

(dimensions: Tableau 1). Cet élargissement de la région frontale se retrouve à des degrés divers chez tous les giraffidés, mais il est ici très accentué. Les orbites sont disposées très obliquement par rapport au plan sagittal, de manière inhabituelle pour un ruminant et même pour un giraffidé. Les ossicônes, dont ne subsistent que les bases, étaient inclinés vers l'arrière, probablement un peu divergents, et insérés nettement en arrière des orbites, leur bord antérieur étant situé au-dessus de la barre post-orbitaire. En arrière, les cornes surplombent nettement la partie antérieure de la fosse temporale. Leur bord latéral, au lieu de se confondre avec le bord latéral du crâne comme chez les Palaeotraginae, est nettement plus médial que le bord orbitaire. Comme elles sont robustes par rapport au frontal, elles viennent presque au contact l'une de l'autre au voisinage du plan sagittal, n'étant séparés que d'environ $15 \mathrm{~mm}$. Par 


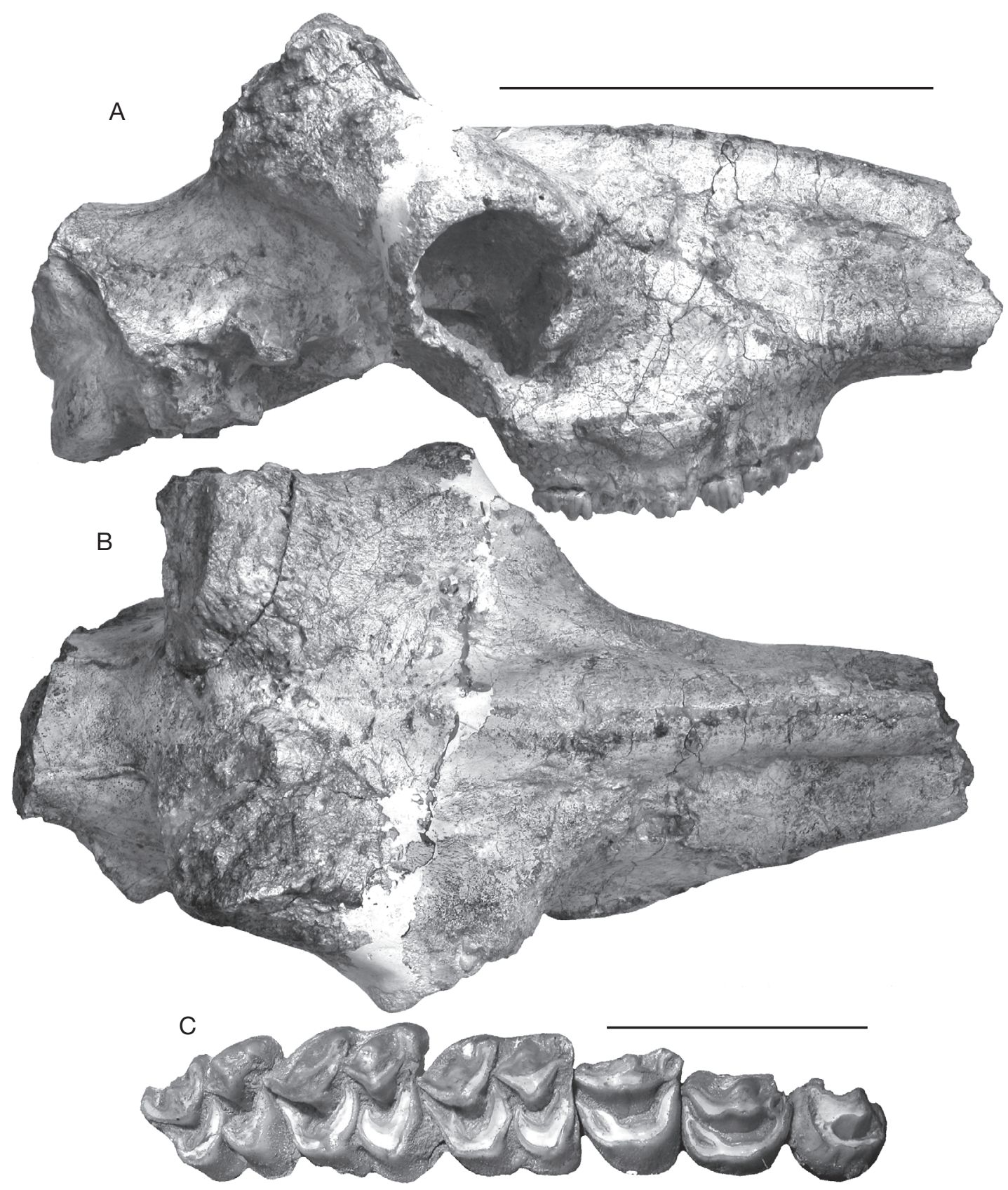

FIG. 2. - Bohlinia attica (Gaudry \& Lartet, 1856) Kirokuçuk: A, crâne A, vue latérale; B, crâne A, vue dorsale; C, série dentaire supérieure droite du crâne B. Échelles: A, B, $20 \mathrm{~cm} ; \mathrm{C}, 5 \mathrm{~cm}$.

rapport à la disposition supraorbitaire primitive chez les ruminants, conservée chez les Palaeotraginae, les cornes ont donc subi un déplacement postéro-médial en lequel Bohlin (1926) avait vu une caractéristique de ce qu'il appelait Giraffinae, ici appelés Giraffini, mais comme on le retrouve 
TABLEAU 2. - Dimensions dentaires (en mm) comparées de Bohlinia attica (Gaudry \& Lartet, 1856) et de la moyenne d'un échantillon $(N=18)$ de Giraffa camelopardalis Linnaeus, 1758 actuelle (valeurs extrêmes entre parenthèses; largeurs des dents d'après Singer \& Boné 1960). Notes: 1, "Camelopardalis vetusta» Wagner, 1861, NHMW; 2, "Orasius atticus» (Gaudry \& Lartet, 1856), Munich; 3, MNHN, Fouilles Arambourg.

\begin{tabular}{lcccc}
\hline & P2-P4 & M1-M3 & largeur P4 & largeur M1 \\
\hline Bohlinia attica, Kirokuçuk A & 60 & 76 & 22,8 & 26 \\
Bohlinia attica, Kirokuçuk B & 61 & 83 & 22,7 & 26,7 \\
Bohlinia attica, Pikermi & - & 79 & 22 & 27 \\
Bohlinia attica, Pikermi2 & c. 59 & c. 76 & 23 & 26 \\
Bohlinia attica, Vathylakkos $^{3}$ & c. 62 & c. 75 & - & - \\
G. camelopardalis & $68(62-74)$ & $88(78-94)$ & $30(26-33)$ & $31(27-35)$ \\
\hline
\end{tabular}

chez Okapia, il caractérise bien les Giraffinae dans leur acception présente. La base des ossicônes est robuste, avec une section quadrangulaire arrondie mais leur surface est imparfaitement préservée ; un peu au-dessus de la base, leur contour devait être à peu près circulaire. Leur surface est finement ridulée, et leur structure interne très compacte. Aucune trace ne subsiste de la suture entre les ossicônes et les os du toit crânien, de sorte que leur nature d'appendices originellement indépendants ne peut être formellement établie, mais leur ressemblance avec les ossicônes de Giraffa et Okapia est telle qu'il est difficile d'imaginer qu'ils ne leur soient pas homologues.

La suture fronto-pariétale est parfaitement soudée, la suture inter-frontale à peine distincte encore, et la fusion de la suture fronto-nasale est aussi bien avancée. On peut penser que cette fusion assez précoce est en rapport avec le mode de combat intra-spécifique, impliquant la rigidité de la boîte crânienne.

Le crâne, à la différence de celui de Giraffa, était certainement peu pneumatisé dans la région supra-orbitaire, le plan dorsal des frontaux en avant des orbites étant à peine plus haut que le bord supérieur de ces dernières (Fig. 2A). Le profil des nasaux est un peu convexe et dans la partie située en avant des orbites, ceux-ci comportent une zone allongée renflée et un peu rugueuse; il n'est pas impossible que cette zone ait pu servir de support à un ossicône médian, comme on en rencontre souvent chez les mâles des girafes actuelles (Spinage 1993 - contra Solounias \& Tang 1990). L'orbite est imparfaitement préservée, de sorte que la forme de son bord ventral (cf. Bohlin 1926: fig. 198) n'est pas déterminable; son bord antérieur est situé à l'aplomb du milieu de M3. Il n'existait sans doute pas de trou lacrymal, comme chez la plupart des Giraffidae, ni de lacune ethmoïdale, qui tend à se combler avec l'âge chez Giraffa. Le trou infraorbitaire est situé un peu en avant de P2, comme chez Giraffa.

Les lignes temporales sont bien nettes en arrière des cornes, où elles sont le plus rapprochées, et s'écartent un peu l'une de l'autre en arrière. L'arrièrecrâne est petit par rapport au reste du crâne, comme il est habituel chez la plupart des Giraffidae, mais les condyles occipitaux sont relativement gros; leur axe est incliné d'environ $40^{\circ}$ par rapport au plan occlusal, de sorte que l'animal pouvait sans doute allonger la tête dans l'axe du cou.

La région auditive est mal conservée, mais il semble que la mastoïde n'ait pas atteint la face ventrale du crâne, comme il est habituel dans cette famille. En vue ventrale, la forte taille des condyles par rapport à la base du crâne est frappante. Le basioccipital comporte des tubérosités antérieures robustes, mais sans doute assez peu saillantes.

Les dents sont petites par rapport au crâne (dimensions: Tableaux 1 et 2), raison pour laquelle plus d'un auteur ont hésité à associer à de semblables dents les os des membres attribués par Gaudry \& Lartet (1856) puis Gaudry (1862-67) à leur Giraffa attica. Ces os sont en effet presque aussi gros que ceux de la girafe actuelle, alors que les dents ne sont guère plus grosses que celles du Palaeotragus rouenii Gaudry, 1861 dont les membres sont bien plus petits.

La morphologie dentaire chez les Giraffidae est peu variable, et ces dents ne peuvent aisément être distinguées de celles de Palaeotragus Gaudry, 1861, 
genre de taille voisine, largement répandu dans le Miocène supérieur de l'Ancien Monde, avec lequel Bohlinia co-existe fréquemment. Les prémolaires sont grosses relativement aux molaires, mais moins que chez les Sivatheriinae; elles sont arrondies, avec des styles assez saillants, et un parastyle dédoublé. Rien en revanche ne distingue les molaires de celles d'un petit Palaeotragus, comme P. rouenii, si ce n'est la taille un peu supérieure.

Le crâne $B$ présente quelques différences avec le crâne A. L'orbite est encore plus antérieure, puisque son bord antérieur atteint le niveau du milieu de M2, et cette différence ne peut être qu'en partie due à l'âge, car cet individu n'est guère plus jeune que le précédent. Les cornes sont situées franchement en arrière des orbites, puisque leur bord antérieur atteint à peine la barre post-orbitaire, et elles étaient aussi bien plus petites, même si leur volume précis ne peut être estimé; il s'agissait donc sans doute d'une femelle.

Comme sur le crâne $\mathrm{A}$, le parastyle des prémolaires est complexe, et enclôt un îlot d'émail (Fig. 2C); il semble qu'il s'agisse donc là d'un bon critère de distinction entre les prémolaires de Bohlinia et celles de Palaeotragus, qui peuvent être par ailleurs très semblables. Les styles et piliers buccaux des molaires sont aussi bien marqués; sur M3 au moins, ils sont plus forts que sur le crâne $A$, à la différence du métastyle de $\mathrm{P} 4$, moins nettement recourbé.

Détaché du crâne, le rocher laisse voir sa partie endocrânienne. Bien qu’imparfaitement conservé, il se distingue bien de celui des girafes actuelles par la convexité de ses deux faces, cérébrale et cérébelleuse, séparées par une arête mousse. Chez G. camelopardalis, les deux faces sont déprimées et séparées par une arête aiguë.

L'espèce est aussi attestée à Karaslari par quelques os des membres incomplètement préparés, deux radius $(\mathrm{L}=760$ et 765$)$ et un métacarpe $(\mathrm{L}=722)$, et par un maxillaire, Ka-2603, comportant P4, M2 et M3 (longueur M1-M3 = c. 77; longueur $\times$ largeur de $\mathrm{P} 4=21,2 \times 23,5)$. Les dents sont brachyodontes, avec de forts styles et piliers labiaux; $\mathrm{P} 4$ possède le parastyle bifide caractéristique de cette espèce.

\section{COMPARAISONS}

Le genre Bohlinia n'est représenté dans le Miocène supérieur de la région balkano-iranienne que par l'espèce Bohlinia attica, assez fréquemment citée, mais dont les crânes sont exceptionnels. Le mieux préservé est un crâne de Pikermi au Naturhistoriska Riksmuseet de Stockholm, que je n'ai pas vu, mais qui a été décrit et figuré par Bohlin (1926: figs 195198 ), et dont D. Kostopoulos a bien voulu me communiquer des photos; il est malheureusement fortement déformé par compression transversale. Les cornes sont encore plus étalées à la base que sur les crânes de Kirokuçuk; leur section est d'abord losangique, comme à Kirokuçuk, mais plus arrondie vers le haut, et l'extrémité est arrondie, mais dépourvue du champignon terminal fréquent chez les mâles actuels.

Un ossicône de Pikermi au NHMW présente une section basale ovale renforcée par un pilier médial ; la surface est parcourue de nombreux petits sillons, et l'extrémité est renflée en champignon. On peut penser que cette différence par rapport au crâne précédent atteste le dimorphisme sexuel, encore que les très longues cornes du crâne de Stockholm ne parlent pas dans ce sens; la variabilité est forte chez la girafe actuelle (Singer \& Boné 1960).

Le genre Bohlinia a été signalé dans plusieurs autres sites de Méditerranée orientale. Dans la région de Thessalonique, plusieurs os des membres du Vallésien du Ravin de la Pluie (Geraads 1979) correspondent par leurs dimensions à ceux du Turolien, et comme aucun autre Giraffidé dolichopode n'est connu dans la région, on peut les rattacher à Bohlinia. Le genre persiste dans les sites du Turolien, jusqu'à Dytiko inclus (Geraads 1979).

Du Vallésien terminal de Nikiti-1, Kostopoulos et al. (1996) ont décrit sous le nom de Bohlinia attica plusieurs crânes, assez complets mais en mauvais état de conservation (NKT-52, NKT145, NKT-148, NKT-172), et sous le nom de B. nikitiae Kostopoulos, Koliadimou \& Koufos, 1996 un arrière-crâne pourvu d'un ossicône (NKT147). La morphologie de cet ossicône indique que B. nikitiae appartient assurément à ce genre, car il ressemble beaucoup à ceux de Pikermi, tant en vue de profil qu'en section, mais l'état de conservation du matériel ne permet guère de pousser bien loin les comparaisons concernant son orientation; la position haute de l'orbite semble bien due à la déformation. L'argumentation de Kostopoulos et al. 
(1996) pour établir leur nouvelle espèce repose surtout sur la comparaison avec les autres crânes du même gisement attribués à $B$. attica, mais ces identifications sont elles-mêmes quelque peu incertaines, en grande partie à cause de la déformation et du mauvais état de conservation du matériel. Il ne fait guère de doute que NKT-172 est en fait un Palaeotragus, comme le montrent ses cornes minces et très écartées l'une de l'autre, et comme le pense maintenant Kostopoulos lui-même (comm. pers. 2008), qui m'en a aimablement communiqué des photos. Les crânes NKT-145 et NKT-52 possèdent des cornes massives et proches l'une de l'autre à la base, comme chez Bohlinia, mais elles semblent moins larges et moins décalées vers l'arrière que dans ce genre. Quoi qu'il en soit, la présence de Bohlinia à Nikiti-1 est indiscutable.

En Afrique, les Giraffini de la deuxième moitié du Pliocène et du Pléistocène sont maintenant rapportés au genre Giraffa (y compris ceux précédemment rattachés à Okapia), mais Likius et al. (2007) ont récemment décrit sous le nom de Bohlinia adoumi un Giraffidae du Miocène terminal des sites de TorosMenalla au Tchad. Cette espèce présente plusieurs particularités par rapport aux formes balkaniques: - alors que chez ces dernières, les cornes tendent vers une forme cylindrique, elles sont ici coniques, bien que moins longues et pointues que chez les Palaeotragus et Samotherium Forsyth-Major, 1888 d'Eurasie; elles rappellent celles du Palaeotragus de Ngorora au Kenya (Hamilton 1978: fig. 38) et celles de Langebaanweg en Afrique du Sud que Harris (1976a) avait d'abord rapportées à Sivatherium, avant de convenir (Harris 1991) qu'elles n'appartenaient pas à ce genre, et qui sont probablement aussi celles d'un paléotraginé;

- à la différence de $B$. attica, rien ne montre que les cornes étaient décalées vers le plan sagittal et l'arrière; aussi bien sur le type que sur un autre spécimen (TM-321-01-01; Likius 2002: fig. 3.19), elles demeurent très éloignées de l'occipital, le diamètre antéro-postérieur basal de la corne étant nettement inférieur à la distance entre celle-ci et la crête occipitale (notons que la mesure 1 du tableau 1 de Likius et al. [2007] ne peut être comparée à celles indiquées par Kostopoulos et al. [1996], qui partent du centre de la corne, et non de son bord postérieur). "Bohlinia» adoumi est donc dépourvu des traits essentiels caractérisant la sous-famille; - les prémolaires inférieures (Likius et al. 2007: fig. 4) sont d'un type banal pour un Giraffidae; elles ne présentent pas l'aspect globuleux de celles des Giraffini, ni la tendance à l'isolement du paraconide de p3-p4 et de l'hypoconide de p3 qu'on note aussi bien chez Giraffa que chez Bohlinia (Bohlin 1926: figs 202, 203).

Bien que le matériel disponible soit insuffisant pour préciser ses affinités, il semble donc bien que le Giraffidae de Toros Menalla soit plus proche des Palaeotraginae que des Giraffini.

Dans le Plio-Pléistocène d'Afrique de l'Est ont été nommées plusieurs espèces de Giraffa, dont la taille varie de celle d'un petit Palaeotragus tel que $P$. rouenii à celle des plus grands $G$. camelopardalis actuels, mais le crâne n'est connu que dans la plus grande forme, G. jumae Leakey, 1967 (voir aussi Leakey 1970). Le frontal est beaucoup moins pneumatisé que chez les girafes actuelles, de sorte que les ossicônes sont moins décalés postérieurement, et le crâne est beaucoup moins surélevé dans la région inter-orbitaire; de plus, il n'existe pas d'ossicône antérieur médian.

Par ces caractères primitifs, G. jumae ressemble à Bohlinia, mais l'espèce africaine est plus proche de G. camelopardalis par la grandeur de ses dents par rapport au crâne, et par la plus grande proximité des cornes à l'occipital. Giraffa jumae diffère de ces deux derniers taxons par ses cornes plus inclinées, mais comme les cornes de G. stillei (Dietrich, 1942), autre espèce du Plio-Pléistocène africain, sont bien redressées (Harris 1987), il semble plutôt que la forte inclinaison des cornes chez $G$. jumae soit un trait dérivé propre à cette espèce. La présence dans le Pliocène de Turquie (Geraads 1998) d'une forme rapprochée de $G$. jumae sur la base d'ossements post-crâniens suggère que l'origine du genre actuel est sans doute à rechercher dans la région égéenne. Quant aux formes indo-pakistanaises, G. punjabiensis Pilgrim, 1910 et G. sivalensis (Falconer \& Cautley, 1843), elles sont trop mal connues pour être utilement discutées.

Boblinia attica diffère de G. camelopardalis par plusieurs caractères du squelette des membres (Geraads 1979) mais il se peut que nombre d'entre eux soient liés à son plus faible poids. Il est 
TABlEAU 3. - Dimensions (en mm) de quelques séries dentaires de Helladotherium Gaudry, 1860 de la République de Macédoine, par comparaison avec quelques autres spécimens du Turolien. Notes: 1, Geraads et al. 2005; 2, Bohlin 1926; 3, PIUW.

\begin{tabular}{lcccc}
\hline & P2-P4 & M1-M3 & largeur P4 & largeur M1 \\
\hline Karaslari 2752 & 97,5 & $120+$ & c. 40 & 41 \\
Kirokuçuk 2737 & 96 & 124 & 42,5 & 42 \\
Vozarci 1870/79 $_{\text {Kalimantsi, Bulgarie1 }}$ & - & - & 42 & 42 \\
Pikermi, Grèce2 $^{2}$ & 95 & 121 & 40,4 & 41,7 \\
Samos, Grèce $^{3}$ & 97 & 120 & 43 & 46 \\
\hline
\end{tabular}

donc tentant de revenir à l'opinion primitive de Gaudry \& Lartet (1856) et d'inclure B. attica dans Giraffa, mais ce rassemblement occulterait d'abord l'étroite parenté qui unit certainement les formes actuelles, ensuite celle qui les unit à $G$. jumae, et sans doute aux autres formes africaines $(G$. pygmaea Harris, 1976, G. stillei). Il vaut donc mieux conserver le nom de Bohlinia pour les formes du Miocène supérieur.

\section{Sous-famille SIVATHERIINAE Murie, 1871}

\section{Genre Helladotherium Gaudry, 1860}

?Bramatherium Falconer, 1845: 363 (espèce type: Bramatherium perimense Falconer, 1845).

ESPÈCE TYPE. - Camelopardalis duvernoyi Gaudry \& Lartet, 1856 par monotypie.

\section{Helladotherium duvernoyi}

(Gaudry \& Lartet, 1856)

Camelopardalis duvernoyi Gaudry \& Lartet, 1856: 274.

TYPe. — Gaudry \& Lartet (1856) n’ont pas désigné d'holotype parmi la série type, ultérieurement mélangée à d'autres spécimens, de sorte qu'il est impossible de désigner un lectotype, mais ceci est sans conséquence, tous les grands Giraffidae de la localité type appartenant à cette espèce.

MATÉRIEL ÉTUdiÉ. - Maxillaire de Karaslari (Ka-2752), base d'un crâne de Kirokucuk (KK-2737), maxillaire de Vozarci (Voz-1870/79) et métacarpien sans numéro du même site. Tout ce matériel est conservé au MHNS.

LOCALITÉ TYPE. — Pikermi (Turolien).
EXTENSION STRATIGRAPHIQUE ET GÉOGRAPHIQUE. Vallésien final au Turolien final de la province balkanoiranienne; le genre a aussi été signalé en France (Gaudry 1873) et en Hongrie (Bernor et al. 2003), mais ces déterminations sont incertaines.

Diagnose. - C'est à cette espèce que sont habituellement attribués les nombreuses dentitions et os des membres de Sivatheriinae du Miocène supérieur de la région balkano-iranienne, mais le crâne du mâle étant toujours inconnu, aucune diagnose ne peut être proposée (voir Discussion de Sivatherium).

\section{DESCRIPTION ET COMPARAISONS}

Un maxillaire de Karaslari (Ka-2752) comporte toutes les jugales supérieures, mais la plus grande partie des murailles externes des molaires manque. Les prémolaires sont grandes relativement aux molaires (dimensions: Tableau 3), P4 est à peu près aussi large que $\mathrm{M} 1$; ces caractères se retrouvent non seulement chez les autres Helladotherium (et Bramatherium), mais aussi chez les autres Sivatheriinae. Ils permettent de les distinguer aisément du paléotraginé Samotherium, qui peut atteindre une taille comparable.

Le spécimen le plus complet est une partie de crâne de Kirokuçuk (KK-2737, Fig. 3I), comportant les dents supérieures et la base du neurocrâne, semblables à ceux du crâne de Pikermi figuré par Gaudry (1862-67). Les dents présentent les mêmes caractères que sur la pièce précédente, $\mathrm{P} 4$ est presque rectangulaire, les molaires ne possèdent que de faibles piliers correspondant au paracône et métacône. La bulle tympanique est très petite, comme chez les Sivatheriinae plio-pléistocènes (Sivatherium), mais la base du crâne est moins raccourcie que chez ces derniers et l'axe du conduit auditif reste oblique, et non transversal (Geraads 1985). Le basioccipital 
TABLEAU 4. - Dimensions (en $\mathrm{mm}$ ) des métacarpiens rapportés à Helladotherium Gaudry, 1860. Abréviations: dist., distal; prox., proximal.

\begin{tabular}{lccc}
\hline & DT prox. & DT dist. & longueur \\
\hline Karaslari & 114 & 111 & 436 \\
Vozarci & 102 & 100 & 408 \\
Pikermi, Grèce & & & \\
$\quad$ BMNH M 11382 & 96 & 92 & 418 \\
BMNH M 11377 & 105 & 103 & 426 \\
MNHN PIK-1526 & 92 & 103 & 410 \\
$\quad$ MNHN PIK-1527 & 97 & 102 & 407 \\
$\quad$ MNHN PIK-1525 & 87 & 97 & 427 \\
Hadjidimovo, Bulgarie & & & \\
$\quad$ sans numero & 118 & 109 & 418 \\
$\quad$ HD-5492 & 115 & 102 & 430 \\
$\quad$ HD-5504 & 110 & 104 & 435 \\
Kalimantsi, Bulgarie & & & \\
$\quad$ sans numero & 125 & 100 & 470 \\
$\quad$ sans numero & 115 & 110 & 435 \\
Maragha, Iran & & & \\
$\quad$ MNHN, sans numero & 92 & $>99$ & 465 \\
\hline
\end{tabular}

se rétrécit rapidement vers l'avant; les tubérosités antérieures sont petites mais bien saillantes.

De Vozarci proviennent un maxillaire avec P3-M2, Voz-1870/79, ainsi qu'un métacarpien semblable par ses proportions à ceux de Pikermi. Un spécimen de Karaslari est un peu plus gros, quoique dans les limites de variation de ceux de Perivolaki (Kostopoulos \& Koufos 2006), ou de ceux de Bulgarie (Geraads et al. 2005), mais plus court et plus massif qu'un métacarpien de Maragha en Iran (dimensions: Tableau 4). Ces différences de proportions s'accordent aussi bien avec la variabilité intraspécifique qu'avec une distinction taxonomique (la forme de Maragha a été distinguée sous le nom de Helladotherium gaudryi Mecquenem, 1924, et rien ne montre même que le Sivatheriinae du site iranien appartienne vraiment à ce genre).

\section{Genre Sivatherium Falconer \& Cautley, 1836}

DIAGNOSE. - Sivatheriinae de très grande taille, à crâne large, donc court et bas. Angle cranio-facial très ouvert, au moins égal à $180^{\circ}$. Base du crâne raccourcie. Une paire de cornes antérieures, situées directement au-dessus des orbites, arrondies, coniques ou aplaties, mais toujours petites et peu ou pas ornementées. Une paire de cornes postérieures, situées très en arrière des orbites, bien écartées l'une de l'autre, divergentes, toujours courbées mais dans des directions variables, au moins un peu aplaties, parcourues de nombreux sillons vasculaires et ornementées le long de leur bord convexe, aminci, de protubérances et rugosités.

ESPÈCE TYPE. - Sivatherium giganteum Falconer \& Cautley, 1836 par monotypie.

\section{Sivatherium garevskii n. sp.}

Holotype. - Fragments d'un crâne comprenant le nasal et une partie du maxillaire, deux dents supérieures, un appendice crânien antérieur et une corne postérieure gauche incomplète, sans numéro, conservés au MHNS (Fig. 3A-G).

ÉTYMOLOGIE. - En reconnaissance de la contribution majeure de Risto Garevski à la paléomammalogie macédonienne.

Localité Type. - Stamer près de Delcevo, nord-est de la République de Macédoine. Le sédiment adhérant aux fossiles ne permet pas de choisir entre la Formation de Pancerevo, attribuée au Miocène supérieur, et celle de Solnje, attribuée au Pliocène (Dumurdzanov et al. 2004); cependant, ce site a livré trois autres fossiles, une P3 référable à Propotamochoerus? sp., genre mio-pliocène, et deux fragments de bois de cervidés n'appartenant sans doute pas à des genres modernes et, bien que très pauvre, un tel assemblage s'accorde mieux avec le Pliocène qu'avec le Miocène supérieur, où les cervidés sont rares et les bovidés dominants.

DiagNOSE. - Sivatherium de taille moyenne d'après ses dents, mais à cornes principales gigantesques, courbées vers l'arrière, presque dépourvues de torsion, mais à bord antérieur formant un bourrelet ondulant, festonné de tubercules rugueux, à paumure atteignant sa largeur maximale vers la mi-longueur. Cornes antérieures petites, formées d'un simple tubercule. Prémolaires modérément développées. Sivatherium garevskii n. sp. se distingue des autres espèces du genre par par la petite taille de ses cornes antérieures, et par la taille beaucoup plus grande des cornes postérieures, dont la paumure s'élargit jusque vers la mi-longueur au lieu de diminuer de la base au sommet.

\section{DESCRIPTION ET COMPARAISONS}

Le genre Sivatherium comprend une forme asiatique, S. giganteum Falconer \& Cautley, 1836 du PlioPléistocène des Siwaliks supérieurs (Indo-Pakistan), surtout connu par un crâne conservé au BMNH (M 15283), et deux formes africaines, S. maurusium (Pomel, 1893) (= S. olduvaiense Hopwood, 1934) 

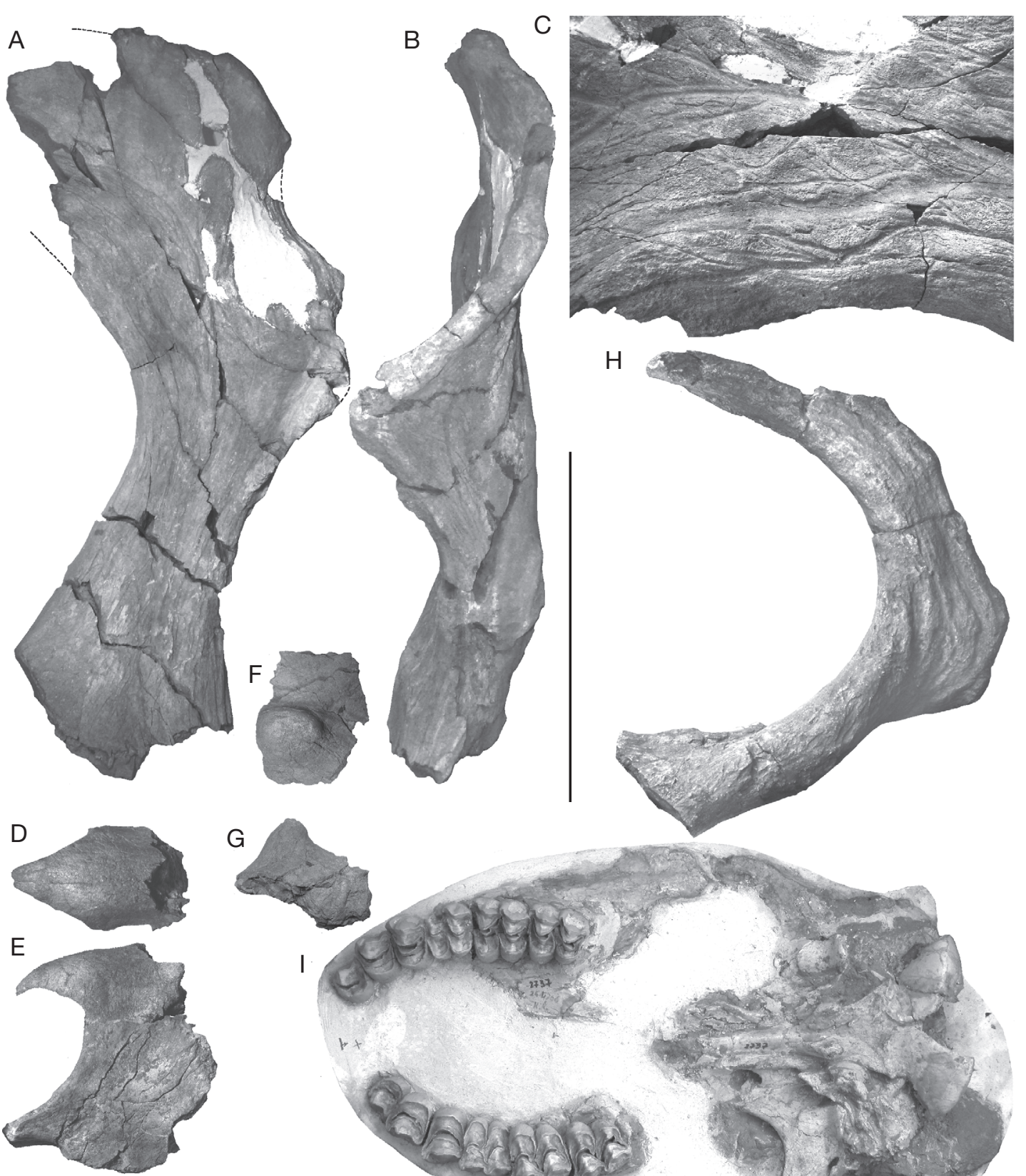

$\mathrm{H}$
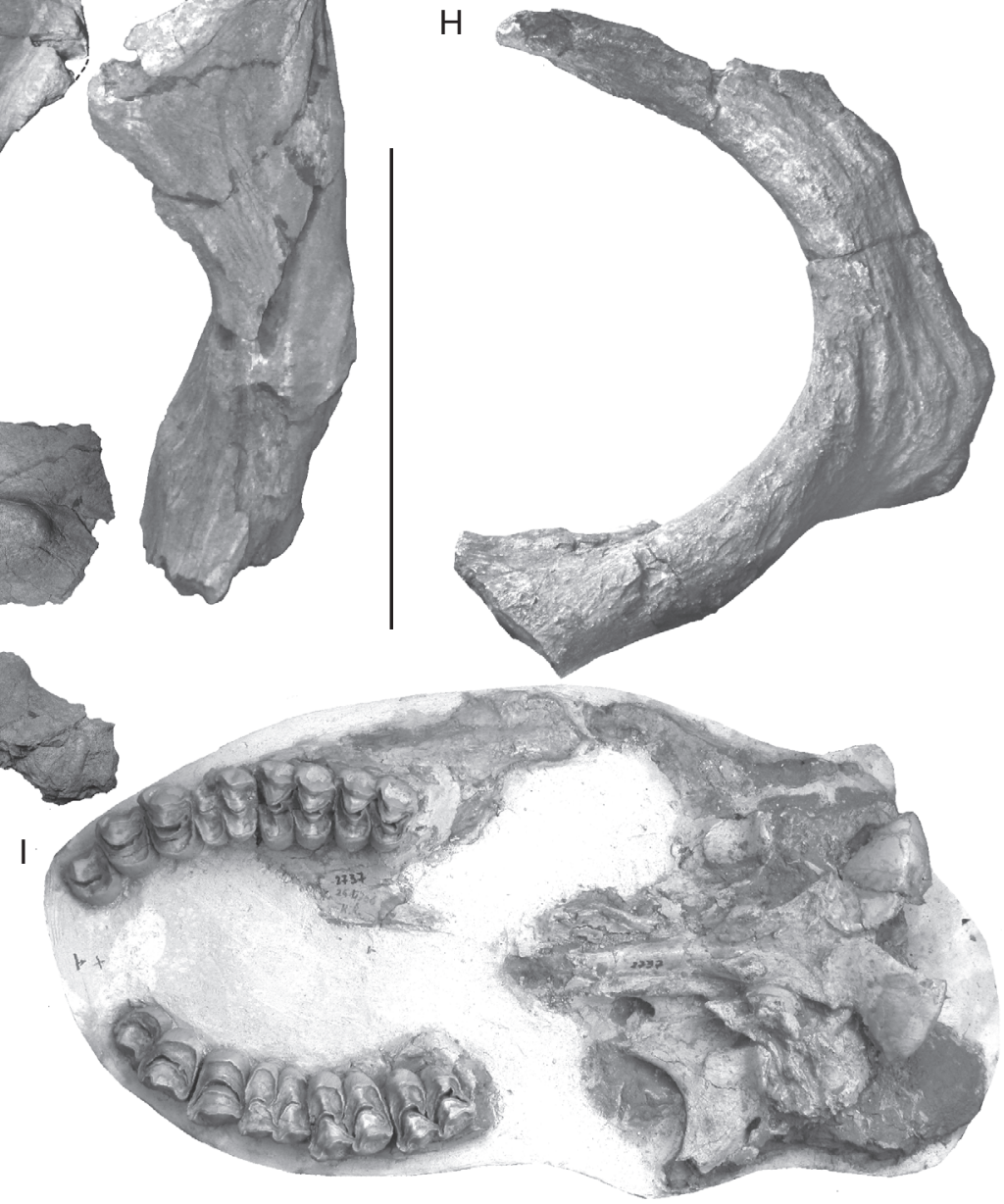

FIG. 3. - A-G, Sivatherium garevskii n. sp., holotype, Stramer près de Delcevo; A-C, corne postérieure gauche; A, vue médio-dorsale; $\mathbf{B}$, vue antérieure ou antéro-latérale; $\mathbf{C}$, détail de la surface pour montrer les sillons vasculaires; $\mathbf{D}$, nasal en vue dorsale; $\mathbf{E}$, reconstruction de la région de l'ouverture nasale en vue latérale; $\mathbf{F}$, G, corne antérieure; $\mathbf{F}$, vue dorsale; G, vue latérale; $\mathbf{H}$, Sivatherium maurusium (Pomel, 1893), Djibouti, vue médio-dorsale du moulage de la corne postérieure à la même échelle qu'A et $B$ pour montrer la taille très inférieure et la torsion; I, Helladotherium duvernoyi, partie inférieure d'un crâne de Kirokuçuk. Échelle: A, B, D-H, 40 cm; $\mathrm{C}, 20 \mathrm{~cm} ; \mathrm{l}, 25 \mathrm{~cm}$. 
du Pliocène moyen et supérieur et du Pléistocène, et $S$. hendeyi Harris, 1976 du Pliocène inférieur de Langebaanweg. La forme macédonienne se distingue bien de ces trois espèces, tant par son origine géographique que par ses caractères crâniens.

Le fragment de museau comporte sans doute la plus grande partie du nasal, basculé à $90^{\circ}$ par rapport au prémaxillaire et à la partie rostrale du maxillaire, la suture entre ces os n'étant pas reconnaissable (Fig. 3D, E). Courts, un peu busqués et pointus en avant, les nasaux sont très semblables à ceux du crâne de $S$. giganteum (BM 15283); Colbert (1935: fig. 172) a représenté les nasaux de Bramatherium, Sivatheriinae plus ancien, sur le même modèle, mais ils sont en fait inconnus. Ceux du S. maurusium de Koobi Fora au Kenya (Harris 1976b: pl. 15) sont également pointus, mais leur profil est presque rectiligne.

Le foramen infra-orbitaire était situé au-dessus de l'alvéole antérieur de P3, un peu plus postérieurement que chez $S$. giganteum et $S$. maurusium (Koobi Fora: Harris 1976b; Djibouti: Geraads 1985). Le fond de l'échancrure nasale se situe à l'aplomb de l'avant de P2, comme sur le specimen de Djibouti ; il semble plus profond sur le specimen de Koobi Fora, et l'est assurément chez $S$. giganteum.

La corne postérieure (Fig. 3A-C) a été remontée à partir de nombreux fragments, mais leurs cassures très fraîches garantissent une reconstruction fidèle. Il manque néanmoins la partie distale, que j'évalue à un tiers au moins de la longueur totale. Son caractère le plus remarquable est sa très grande taille, dépassant nettement celle de tous les autres Giraffidae, y compris les plus grands Sivatherium; chacune devait peser autour de $20 \mathrm{~kg}$ sur le vivant.

L'absence de torsion ne permet pas d'orienter la corne sans ambiguïté, mais à la base, la face la plus plane doit correspondre à la face médiale (dorsale), et il s'agit donc très probablement d'une corne gauche. La corne est donc recourbée vers l'arrière. Elle est fortement comprimée transversalement, le rapport entre son diamètre antéro-postérieur et le diamètre transversal (épaisseur) variant entre 1,4 au niveau de la constriction peu au-dessus de la base, et environ 4 au niveau où la corne est la plus étalée. Le bord postérieur (concave) est épais, de $10 \mathrm{~cm}$ au maximum près de la base, cette épaisseur dimi- nuant lentement vers le haut. La section transversale montrerait néanmoins, au dessus de la constriction où cette section est à peu près ovale, une diminution assez rapide de cette épaisseur vers le centre de la corne, de sorte que la plus grande partie est assez mince, l'épaisseur minimum étant d'environ $25 \mathrm{~mm}$. Le bord antérieur est néanmoins un peu épaissi (environ 4 à $5 \mathrm{~cm}$ ); il porte de plus des rugosités et nodosités irrégulières, qui contribuent à accentuer les variations importantes de l'étalement de la corne. Au-dessus de la constriction située peu au-dessus de la base, où la DAP n'est que de 140 $\mathrm{mm}$, la corne s'étale en effet largement puisque cette DAP double, pour diminuer un peu, puis augmenter à nouveau (DAP maximale $=310 \mathrm{~mm}$ ). Bizarrement, la DAP ne diminue donc pas plus ou moins régulièrement comme chez les Sivatherium africains, mais la paumure s'élargit au contraire vers le haut, comme chez le daim. Il est néanmoins presque certain que l'étalement diminuait dans la partie terminale manquante.

$\mathrm{Si}$, en vue médio-dorsale (Fig. 3A), la corne semble à peu près plane, la vue antéro-latérale (Fig. 3B) montre que ce bord est affecté par une gracieuse ondulation qui le porte d'abord franchement en avant, puis le ramène dans le plan principal, puis le porte à nouveau un peu vers l'avant. Chez $S$. maurusium, au contraire, cette bordure décrit habituellement une courbe régulière, hétéronyme (Fig. $3 \mathrm{H}$; Singer $\&$ Boné 1960: pl. 1a, c, d, e, pl. 3, pl. 29c, d; Harris 1974: fig. 4; 1991: fig. 4.25 Arambourg 1979: pl. 27, fig. 3, pl. 28, figs 6, 7 ; Geraads 1985: fig. 1). Dans de rares cas, la corne peut être dépourvue de torsion (Singer \& Boné 1960: pl. 1b, f; type C de Harris 1974), mais dans tous les cas la paumure se réduit vers le haut. Les cornes de $S$. hendeyi du Pliocène inférieur de Langebaanweg (Harris 1976a) sont recourbées mais simples, presque dépourvues de tubérosités, et à peine aplaties. Malgré un âge peut-être voisin, elles sont donc très différentes de celles de $S$. garevskii.

Les cornes postérieures de $S$. giganteum sont mal connues. Falconer \& Cautley (1836) n'en ont figuré que des fragments, le plus complet (Murchison 1868: pl. 21, fig. 3, BMNH M 39525) étant une corne gauche, et supposée comme telle par ces auteurs et la plupart des auteurs ultérieurs, mais 
comme la figure est inversée, la reconstitution du crâne de $S$. giganteum était fausse, les cornes droite et gauche étant interverties, comme l'a noté Abel (1904). On y retrouve l'ondulation du bord antérieur, mais il existe en plus un fort "andouiller" postéro-externe (et non postéro-interne comme le montrent les figures de Falconer \& Cautley 1836, et de Colbert 1935), totalement absent à Stamer et en Afrique. Un autre spécimen, figuré par Colbert (1935: fig. 171), comporte aussi des excroissances situées à l'opposé l'une de l'autre.

En Europe, le seul spécimen comparable à celui de Stamer est un fragment de corne de Turquie d'Europe, d'âge incertain mais sans doute pliocène, attribué par Abel (1904) à Sivatherium, et conservé au PIUW. Il ne s'agit que d'un tronçon, mais il correspond assez bien à celui de Stamer, malgré une courbure vers l'intérieur du bord postérieur. Les proximités géographique et, peut-être, chronologique, accroissent la probabilité qu'il s'agisse d'une même espèce.

L'absence d'érosion sur la plus grande partie de la corne de Stamer permet d'observer en détail les reliefs de sa surface (Fig. 3C). Aucune zone n'est lisse. Le bord antérieur est rugueux, mais tout le reste de la surface est couvert de sillons de largeur variable. Singer \& Boné (1960: 495, 496) contestaient qu'il puisse s'agir d'empreintes vasculaires parce que leur diamètre ne diminue pas de la base au sommet, qu'elles ne sont pas liées aux foramens nourriciers, qu'elles ne sont pas plus fortes que sur les cornes antérieures qui sont bien plus petites, et que de tels vaisseaux seraient dangereusement exposés. Ces auteurs supposaient qu'ils auraient pu en fait servir d'ancrage à des attaches ligamentaires ou cartilagineuses d'un étui corné; cette hypothèse semble avoir été acceptée par Harris (1991: 117), mais elle est invraisemblable. Tout d'abord, la corne de Stamer montre bien qu'aucun des sillons présents vers l'extrémité supérieure n'atteint la taille des plus gros de ceux de la base; leur diamètre moyen diminue, même si d'apparentes anastomoses ne permettent pas de les suivre sur toute leur longueur. L'appendice crânien antérieur de Stamer ne porte que des sillons bien plus petits. Quant au risque d'exposer ces vaisseaux superficiels, les cervidés montrent qu'il est limité; il est probable que ces vaisseaux ne persistaient que pendant la phase de croissance de la corne, laissant ensuite, comme chez les cervidés, l'os à nu. La morphologie complexe de la corne et la présence de rugosités sur le bord antérieur excluent tout revêtement kératinisé.

Quelques fragments du toit crânien ont également été retrouvés, mais je ne suis pas parvenu à établir le contact entre la corne postérieure et la petite corne antérieure. Cette dernière forme un simple tubercule un peu allongé (antéro-postérieurement?), d'environ $85 \times 75 \mathrm{~mm}$ pour $50 \mathrm{~mm}$ de hauteur (mesures approximatives, les limites de la corne étant très vagues).

La taille et la morphologie des appendices crâniens antérieurs different aussi de celles des autres espèces. Chez $S$. giganteum, ils méritent le nom de cornes, étant beaucoup plus longs, aplatis à la base mais coniques vers la pointe. Les appendices antérieurs de $S$. hendeyi, s'ils existent, sont inconnus. Harris (1974) avait attribué à cette espèce une corne longue et conique qui appartient sans doute en fait à un autre taxon, comme noté plus haut. Chez $S$. maurusium les appendices antérieurs sont aplatis, allongés, et peuvent être arrondis en vue latérale (Geraads 1985) ou ensellés (Singer \& Boné 1960: pl. 29a, b; Harris 1991: fig. 4.20). On peut exclure l'éventualité que ces différences soient de nature ontogénique, car les dents de Stamer sont très usées, ou sexuelle, car les cornes postérieures du type de $S$. garevskii sont trop robustes pour être celles d'une femelle dont l'ornementation crânienne serait moins développée que chez les mâles; il se peut que les femelles aient été inermes, comme chez $S$. giganteum.

La dentition n'est représentée que par un fragment de molaire et une prémolaire, toutes deux très usées. Les dimensions de la prémolaire $(\mathrm{L}=$ 32 , largeur $=42$ ), probablement une P4, sont celles d'Helladotherium (Bohlin 1926) ou de S. maurusium (Geraads 1996: tab. 1). La longueur de la rangée des prémolaires devait être inférieure à $100 \mathrm{~mm}$; elle était donc plus courte que chez certains individus de cette dernière espèce, formes géantes récentes d'Afrique du Sud, ou au contraire formes les plus anciennes de Langebaanweg (Harris 1976a) ou de Tunisie (Arambourg 1979), et nettement plus courte aussi que chez $S$. giganteum. Ces dimensions 
modérées de la dentition contrastent avec le gigantisme des cornes.

\section{DisCUSSION}

La corne de S. garevskii de Stamer n'est que la seconde corne de Sivatheriinae découverte en Europe, plus d'un siècle après la première (Abel 1904). Elle complique un peu la question de la nature réelle de l'Helladotherium, connu depuis plus de 150 ans dans le Miocène supérieur, ou tout au moins le Turolien, de la région égéenne, à la suite des travaux de Gaudry \& Lartet (1856) et de Gaudry (1862-67) sur le matériel de Pikermi, mais dont le seul crâne adulte connu reste celui, inerme, décrit de ce site par ces auteurs. Puisque tous les autres Sivatheriinae, tant asiatiques qu'africains, possèdent des appendices crâniens bien développés, il est clair que le crâne de l' "Helladotherium» est celui d'une femelle d'un autre genre, dont la nature demeurait inconnue, les nombreuses fouilles menées dans le Miocène supérieur de la région égéenne n’ayant livré aucune corne (on peut soupçonner que si les restes d'appendices crâniens ont si rarement été signalés, c'est qu'ils sont sans doute bien peu reconnaissables sur le terrain, et que plus d'un ont été détruits au cours des fouilles). Â la suite de la description d'un crâne juvénile du Miocène supérieur de Kavakdere en Anatolie, Geraads \& Güleç (1999) ont conclu qu'il s'agissait sans doute de Bramatherium, genre jusque là limité aux péninsules indienne et arabique. Le crâne de Bramatherium, décrit et figuré par Bettington (1846), Colbert (1935) et Lewis (1939), est plus extraordinaire encore que celui de Sivatherium, puisqu'il présente deux paires de cornes, supra-occipitales et fronto-pariétales, ces dernières fusionnées à la base chez l'adulte, et dirigées presque verticalement. On peut néanmoins fort bien concevoir que le crâne de la femelle en ait été dépourvu, et de ce fait, attribué à un genre différent. Bramatherium - Helladotherium serait donc l'un des rares genres de Ruminants (avec par exemple les Bovidae Tragoportax Pilgrim, 1937 et Nisidorcas Bouvrain, 1979) communs à la région balkanique et au sous-continent indien au Miocène supérieur.

La confirmation, à la suite d'Abel, de la présence d'un autre sivatheriiné en Europe orientale, $S$. garevskii, soulève maintenant la possibilité que
Helladotherium soit en fait plus voisin de Sivatherium. Bohlin (1926) avait montré que la femelle de $S$. giganteum est un autre "genre" asiatique, Indratherium, distinct d' Helladotherium, mais ceci ne permet pas d'exclure que ce dernier soit la femelle de $S$. garevskii. Cette hypothèse me semble néanmoins peu probable, parce que le giraffidé de Stamer est sans doute plus récent que Helladotherium, et surtout parce que Bramatherium est un candidat bien plus satisfaisant à ce poste (Geraads \& Güleç 1999).

\section{CONCLUSION}

Le matériel du Miocène supérieur conservé à Skopje ne comprend donc que deux Giraffidae, Helladotherium duvernoyi et Bohlinia attica. Aucun reste ne peut être rapporté à Palaeotragus rouenii, espèce pourtant signalée à Veles par Schlosser (1921), et probablement correctement identifiée par cet auteur, qui la décrivit cependant aussi sous le nom de Camelopardalis parva. Notons néanmoins que le matériel de Veles décrit par Schlosser provenait d'un site (Prevalets) très peu exploité par R. Garevski. Ciric (1957), en revanche, ne signalait que Helladotherium. Aucun reste non plus ne peut être rattaché à Samotherium.

Les Giraffidae du Miocène supérieur de la République de Macédoine sont trop peu abondants pour qu'on puisse en tirer beaucoup de conclusions biochronologiques ou biogéographiques. Tout au plus peut-on noter que les espèces présentes rappellent plus Pikermi, Kerassia (Roussiakis \& Iliopoulos 2004), Nikiti (Kostopoulos et al. 1996) ou les sites bulgares de Kalimantsi et Hadjidimovo (Geraads et al. 2005) que ceux de la basse vallée de l'Axios (Geraads 1978, 1979; Bonis \& Bouvrain 2006), pourtant situés dans le même bassin, où "Helladotherium» n'est pas connu avec certitude mais où sont présents d'autres genres, dont Samotherium par ailleurs rarissime en Europe

Quant à $S$. garevskii, probablement pliocène, il est manifestement voisin, tant des formes africaines de Sivatherium, que de S. giganteum des Siwaliks supérieurs. Si au Miocène et au Pliocène, l'existence de connections entre Afrique et Asie du Sud est bien 
documentée, les formes communes à l'Afrique et aux régions septentrionales, en particulier la région balkano-iranienne, sont plus rares. Il faut surtout rappeler la mention, chez les giraffidés, de Giraffa cf. jumae, espèce africaine, dans le Pliocène de Çalta en Turquie (Geraads 1998). Parmi les autres Ruminants, on ne peut guère citer que Tragoportax, de très vaste répartition géographique, et quelques rares formes comme Dytikodorcas, présent en Grèce et en Libye (Bouvrain \& Bonis 2007), ou Budorcas, récemment signalé en Ethiopie (Gentry 1996).

\section{Remerciements}

C'est Louis de Bonis qui a, le premier, suscité mon intérêt pour les giraffidés fossiles, mais je lui dois bien plus, puisqu'il a guidé mes premiers pas de paléontologue, tant au regretté Laboratoire de $\mathrm{Pa}$ léontologie des vertébrés et Paléontologie humaine de l'Université Paris 6 que sur le terrain, en France et en Grèce. Par la suite, d'autres campagnes de fouilles communes, en Turquie ou à Djibouti, m'ont confirmé à quel point la qualité de vie pendant et après la fouille pouvaient heureusement influencer l'enthousiasme et l'efficacité des participants.

Je remercie également R. Garevski qui a bien voulu m'autoriser à travailler sur les collections qu'il a récoltées, en compagnie de N. Spassov grâce à qui nous avons pu effectuer plusieurs missions en République de Macédoine. Ce travail a bénéficié des remarques détaillées et pertinentes d'un rapporteur anonyme, de A. Ohler et de D. Kostopoulos (Université de Thessalonique); ce dernier m'a également aimablement fourni des documents inédits sur les giraffidés de Nikiti et de Pikermi, et je dois à G. Merceron (Université de Lyon) plusieurs renseignements sur ceux de Vienne (NHMW et PIUW). Merci aussi à A.W. Gentry pour son aide sur la littérature ancienne.

\section{RÉFÉRENCES}

Abel O. 1904. - Über einen Fund von Sivatherium giganteum bei Adrianopel. Sitzungsberichte der Mathematisch Naturwissenschaftlichen Klasse der kaiserlichen Akademie der Wissenschaften 113: 1-22.

ARAMBOURG C. 1979. - Vertébrés villafranchiens d'Afrique $d u$ Nord. Fondation Singer-Polignac, Paris, 141 p.
Bernor R. L., Feibel C. \& Viranta S. 2003. - The vertebrate locality of Hatvan, late Miocene (Middle Turolian, MN12), Hungary, in PeTCulescu A. \& STIUCA E. (eds), Advances in Vertebrate Paleontology "Hen to Panta". A tribute to Constantin Radulescu and Petre Mihai Samson. Académie roumaine, Bucarest: 105-111.

Bettington A. 1846. - Memorandum on certain fossils, more particularly a new Ruminant found at the island of Perim, in the gulf of Cambay. Journal of the Royal Asiatic Society of Great Britain and Ireland 8: 340-348; 417-419.

Bohlin B. 1926. — Die Familie Giraffidae. Paleontologia Sinica C, 4 (1): 1-178.

Bonis L. DE \& Bouvrain G. 2006. - Nouveaux Giraffidae du Miocène supérieur de Macédoine (Grèce), in Petculescu A. \& Stiuca E. (eds), Advances in Vertebrate Paleontology "Hen to Panta". A tribute to Constantin Radulescu and Petre Mihai Samson. Académie roumaine, Bucarest: 5-16.

Bouvrain G. \& Bonis L. DE 2007. - Ruminants (Mammalia, Artiodactyla: Tragulidae, Cervidae, Bovidae) des gisements du Miocène supérieur (Turolien) de Dytiko (Grèce). Annales de Paléontologie 93: 121-147.

Brown D. M., Brenneman R. A., Koepfli K.-P., Pollinger J. P., Milá B., Georgiadis N. J., Louis E. E. Jr, Grether G. F., Jacobs D. K. \& Wayne R. K. 2007. - Extensive population genetic structure in the giraffe. BMC Biology 5: 57.

CIRIC A. 1957. - Pikermiska fauna iz okoline Titovog Velesa. Bulletin du Muséum d'Histoire naturelle du Pays serbe A 8: 1-82.

COLBERT E. H. 1935. - Siwalik Mammals in the American Museum of Natural History. Transactions of the American Philosophical Society 26: 1-401.

Dumurdzanov N., Serafimovski T. \& Burchfiel B. C. 2004. - Evolution of the Neogene-Pleistocene basins of Macedonia. Geological Society of America Digital Maps and Chart Series 1: 1-20.

FALCONER H. 1845. - Description of some fossil remains of Dinotherium, giraffe and other Mammalia, from the Gulf of Cambay, western coast of India. Quarterly Journal of the Geological Society of London 1: 356-372.

Falconer H. \& Cautley P. T. 1836. - Sivatherium giganteum, a new fossil Ruminant genus from the valley of Markanda, in the Siwalik branch of the SubHimalayan mountains. Asiatic Researches 19: 1-24.

ForSTEN A. \& GAREVSKI R. 1989. - Hipparions (Mammalia, Perissodactyla) from Macedonia (Yugoslavia). Geologica Macedonica 3 (2): 159-206.

GAREVSKI R. 1960a. - Neuer Fund von Mastodon in den Diatomeenschichten bei Barobo (Kavadarci), Mazedonien. Fragmenta balcanica Musei Macedonici Scientiarum Naturalium 3 (16): 133-144.

GAREVSKI R. 1960b. — Die Mastodonreste beim Bahnhof 
Čaška in Mazedonien. Acta Musei Macedonici Scientiarum Naturalium 7 (4): 75-87.

GAREVSKI R. 1974a. - Beitrag zur Kenntniss der Pikermifauna Mazedoniens. Fossilreste der Simocyoniden. Fragmenta balcanica Musei Macedonici Scientiarum Naturalium 9 (19): 189-197.

GAREVSKI R. 1974b. - Beitrag zur Kenntnis der Pikermifauna Mazedoniens - Fossilreste der Chalicotheriiden. Fragmenta Balcanica 9 (20): 189-197.

GAREVSKI R. 1976. — Eine weiterer Beitrag zur Kenntnis der Pikermifauna Mazedoniens. Die Dinotherienreste von der Umgebung von T. Veles. Fragmenta Balcanica Musei Macedonici Scientiarum Naturalium 10 (3): 21-25.

GAREVSKI R. 1989. - Ein Aceratherium Schädel von dem Dorf Karaslari in der Umgebung von Titov Veles (Mazedonien). Geologica Macedonica 4 (1): 133-150.

GAREVSKI R. \& Zapfe H. 1983. - Weitere Chalicotheriiden-Funde aus der Pikermi-Fauna von Titov Veles (Mazedonien, Jugoslawien). Acta Musei Macedonici Scientiarum Naturalium 17 (1): 1-20.

GAUDrY A. 1862-67. - Animaux fossiles et Géologie de l'Attique. F. Savy, Paris, 2 vols, 472 p., 75 pls.

GaUdry A. 1873. - Animaux fossiles du Mont Léberon (Vaucluse). F. Savy, Paris, 112 p.

GAUDRY A. \& LARTET E. 1856. - Résultats des recherches paléontologiques entreprises dans l'Attique sous les auspices de l'Académie. Comptes-rendus hebdomadaires des séances de l'Académie des Sciences 43: 271-274.

GENTRY A. W. 1996. - A fossil Budorcas from Africa, in SteWART K. M. \& Seymour K. L. (eds), Palaeoecology and Palaeoenvironments of Late Cenozoic Mammals, Tributes to the career of C.S. (Rufus) Churcher. University of Toronto Press, Toronto: 571-587.

GENTRY A. W. 2003. - Ruminantia (Artiodactyla), in Fortelius M., Kappelman J., Sen S. \& Bernor R. L. (eds), Geology and Paleontology of the Miocene Sinap Formation, Turkey. Columbia University Press, New York: 332-379.

GeraAds D. 1978. — Les Palaeotraginae (Giraffidae, Mammalia) du Miocène supérieur de la région de Thessalonique (Grèce). Géologie méditerranéenne 5 (2): 269-276.

GerAadS D. 1979. - Les Giraffinae (Artiodactyla, Mammalia) du Miocène supérieur de la région de Thessalonique (Grèce). Bulletin du Muséum national d'Histoire naturelle C, 1 (4): 377-389.

GERAADS D. 1985. - Sivatherium maurusium (Pomel) (Giraffidae, Mammalia) du Pléistocène de la République de Djibouti. Paläontologische Zeitschrift 59 (3/4): 311-321.

GERAADS D. 1986. - Remarques sur la systématique et la phylogénie des Giraffidae (Artiodactyla, Mammalia). Geobios 19 (4): 465-477.

GERAADS D. 1991. - Derived features of Giraffid ossi- cones. Journal of Mammalogy 72 (1): 213-214.

GERAADS D. 1996. - Le Sivatherium (Giraffidae, Mammalia) du Pliocène final d'Ahl al Oughlam (Casablanca, Maroc), et l'évolution du genre en Afrique. Paläontologische Zeitschrift 70 (3/4): 623-629.

GERAADS D. 1998. — Le gisement de vertébrés pliocènes de Çalta, Ankara, Turquie. 9. Cervidae et Giraffidae. Geodiversitas 20 (3): 455-465.

GERAADS D. \& GÜLEC E. 1999. - A Bramatherium skull (Giraffidae, Mammalia) from the upper Miocene of Kavakdere (Central Turkey). Biogeographic and phylogenetic implications. Bulletin of the Mineral Research and Exploration 121: 51-56.

GeraAdS D., Spassov N. \& Kovachev D. 2005. — Giraffidae (Artiodactyla, Mammalia) from the late Miocene of Kalimantsi and Hadjidimovo, South-Western Bulgaria. Geologica Balcanica 35 (1-2): 11-18.

Geraads D., Spassov N. \& Garevski R. 2008. — New specimens of Propotamochoerus (Suidae, Mammalia) from the late Miocene of the Balkans. Neues Jahrbuch für Geologie und Paläontologie 248(1): 103-113.

Hamilton W. R. 1978. — Fossil Giraffes from the Miocene of Africa and a revision of the phylogeny of the Giraffoidea. Philosophical Transactions of the Royal Society of London 283: 165-229.

HARRIS J. M. 1974. - Orientation and variability in the ossicones of African Sivatheriinae (Mammalia: Giraffidae). Annals of the South African Museum 65 (6): 189-198.

HARRIS J. M. 1976a. — Pliocene Giraffoidea (Mammalia, Artiodactyla) from the Cape Province. Annals of the South African Museum 69 (12): 325-353.

Harris J. M. 1976b. - Pleistocene Giraffidae (Mammalia, Artiodactyla) from East Rudolf, Kenya. Fossil Vertebrates of Africa 4: 283-332.

Harris J. M. 1987. — Fossil Giraffidae and Camelidae from Laetoli, in LeAKEY M. D. \& HARRIS J. M. (eds), Laetoli, a Pliocene site in Northern Tanzania. Clarendon Press, Oxford: 358-377.

Harris J. M. 1991. - Family Giraffidae, in Harris J. M. (ed.), Koobi Fora Research Project. Volume 3: The fossil ungulates: Geology, Fossil artiodactyls and palaeoenvironments. Clarendon Press, Oxford: 93-138.

Kostopoulos D. S., Koliadimou K. K. \& Koufos G. D. 1996. - The giraffids (Mammalia, Artiodactyla) from the late Miocene mammalian localities of Nikiti (Macedonia, Greece). Palaeontographica 239 (1-3): 61-88.

Kostopoulos D. S. \& Koufos G. D. 2006. - The late Miocene vertebrate locality of Perivolaki, Thessaly, Greece. 8. Giraffidae. Palaeontographica 276 (1-6): 135-149.

LEAKEY L. S. B. 1970. — Additional information on the status of Giraffa jumae from East Africa. Fossil Vertebrates of Africa 2: 325-330.

LEWIS G. E. 1939. - A new Bramatherium Skull. Ame- 
rican Journal of Science 237 (4): 275-280.

Likius A. 2002. - Les grands ongulés du Mio-Pliocène du Tchad (Rhinocerotidae, Giraffidae, Camelidae): systématique, implications paléobiogéographiques et paléoenvironnementales. Thèse, Université de Poitiers, France, 193 p.

Likius A., Vignaud P. \& Brunet M. 2007. — Une nouvelle espèce du genre Bohlinia (Mammalia, Giraffidae) du Miocène supérieur de Toros-Menalla, Tchad. Comptes rendus Palevol 6: 211-220.

MurCHISON C. 1868. - Palaeontological Memoirs and Notes of the Late Hugh Falconer, A.M., M.D. Vol.1. Fauna Antiqua Sivalensis. Hardwicke, London, 590 p.

RousSiAKIs S. J. \& Iliopoulos G. 2004. — Preliminary observations on the metrical variation of Helladotherium duvernoyi and Bohlinia attica. 5th International Symposium on Eastern Mediterranean Geology, Thessaloniki: 343-346.

SCHLOSSER M. 1921. - Die Hipparionenfauna von Veles in Mazedonien. Abhandlungen der Bayerischen Akademie der Wissenschaften Mathematisch-naturwis- senschaftliche Klasse 29 (4): 3-54.

Singer R. \& BONÉ E. L. 1960. - Modern giraffes and the fossil Giraffids of Africa. Annals of the South African Museum 45 (4): 375-548.

SOLOUNIAS N. 2007. - Family Giraffidae, in Prothero D. \& Foss S. E. (eds), The Evolution of Artiodactyls. Johns Hopkins University Press, Baltimore: 257-277.

SOLOUNIAS N. \& TANG N. 1990. - The two types of cranial appendages in Giraffa camelopardalis (Mammalia, Artiodactyla). Journal of Zoology 222: 293-302.

Spassov N. \& GeraAds D. 2004. - Tragoportax Pilgrim, 1937 and Miotragocerus Stomer, 1928 (Mammalia, Bovidae) from the Turolian of Hadjidimovo, Bulgaria and a revision of the late Miocene Mediterranean Boselaphini. Geodiversitas 26 (2): 339-370.

Spinage C. A. 1993. - The median ossicone of Giraffa camelopardalis. Journal of Zoology 230: 1-5.

Thenius E. 1992. - Das Okapi (Mammalia, Artiodactyla) von Zaire - "lebendes Fossil" oder sekundäre Urwaldbewohner? Zeitschrift für zoologische Systematik und Evolutions-Forschung 30: 163-179.

Soumis le 8 décembre 2008; accepté le 20 octobre 2009. 